\title{
Inflation in curved model universes with non-critical density
}

\author{
Peter Hübner and Jürgen Ehlers \\ Max-Planck-Institut für Physik und Astrophysik, Institut für Astrophysik, Karl- \\ Schwarzschildstrasse 1, D-8046 Garching, Federal Republic of Germany
}

Recejved 17 August 1989, in final form 27 July 1990

\begin{abstract}
Non-flat Friedmann-Lemaître models solving the horizon problem in which, prior to and during inflation, curvature, cosmological constant and radiation are taken into account, are constructed for present density parameter values in the range $0.3 \leqslant \Omega_{0} \leqslant 1.5$. The presentation is based on an exact solution to the Lemaître equation containing the three above-mentioned contributions. Then the influence of matter different from radiation is discussed. We also give an example for what happens when the expansion is anisotropic.
\end{abstract}

\section{Introduction}

The large-scale structure of the universe is successfully represented by a RobertsonWalker ( $R W$ ) spacetime filled with matter and radiation obeying Einstein's field equation. Such models, being based on the a priori assumptions of spatial homogeneity and isotropy, cannot explain these two fundamental properties which are well supported particularly by the observed isotropy of the $3 \mathrm{~K}$ background radiation. Even if one assumes homogeneity and isotropy of spacetime, an explanation of the corresponding properties of matter and radiation in terms of transport processes fails because the comoving radius $u_{\mathrm{d}}$ of the particle horizon at the time of decoupling $t_{\mathrm{d}}$, the 'primeval particle horizon', is much smaller than the comoving radius $u_{\mathrm{v}}$ of that part of the universe which became visible at decoupling, the "visual horizon'.

Elementary particle theories assume the existence of scalar fields in the very early phases of the universe. These scalar fields can simulate a cosmological constant [1]. If these scalar fields decay long before decoupling, the horizon problem can be solved, as first pointed out by Guth [2]. The solution of the horizon problem and related problems make inflationary cosmology attractive.

It has been claimed that inflation yields a very small spatial curvature. In fact most calculations in inflationary cosmology have been done with a flat $\mathrm{R} w$ metric. Also it has been predicted that this vanishing spatial curvature can be used to test inflation $[3,4]$. Ellis has shown, however, with a simple model that it is possible to solve the horizon problem in cosmological models with an inflationary phase and to get a non-negligible curvature today. In these models the present value of the density parameter $\Omega=\kappa \rho_{\text {tot }} / 3 H^{2}$ does not have to equal 1 to high accuracy, either. Below we present an improved model supporting the essential conclusions of Ellis. Our model takes into account the effects of radiation and curvature at all times. Moreover, we avoid the unphysical discontinuity of source terms at the onset of inflation used by Ellis for computational simplicity. In contrast to Ellis", our model yields only a lower bound for the expansion during the inflationary phase. If used as a background model for fluctuations of the scalar field, it can therefore be adjusted even if the fluctuation calculations lead to larger lower bounds for the amount of inflationary expansion. 
We also consider deviations from the idealizations of our two-phase model. These deviations can significantly modify the predictions of an inflationary model. It would be very important to know whether inflation works in general, inhomogeneous models of the universe. Recent numerical studies by Goldwirth and Piran indicate that "a region of several horizon sizes must be homogeneous for inflation to begin' [5].

The merit of the idea of inflation, its explanatory and predictive power, thus requires further scrutiny.

\section{Ellis' model}

In this section we briefiy review the model of Ellis [6] for comparison with the two-phase model to be treated in section 3. We put $c=1$ and $\kappa=8 \pi G, G$ denoting Newton's constant.

The evolution of a Friedmann-Lemaitre universe with metric

$$
\begin{aligned}
& \mathrm{d} s^{2}=-\mathrm{d} t^{2}+R^{2}(t)\left(\mathrm{d} \psi^{2}+S_{h}^{2}(\psi)\left(\mathrm{d} \theta^{2}+\sin ^{2} \theta \mathrm{d} \phi^{2}\right)\right) \\
& S_{h}(t)= \begin{cases}\sinh \psi & k=-1 \\
\psi & k=0 \\
\sin \psi & k=1\end{cases}
\end{aligned}
$$

is divided into the three phases indicated in figure 1.

An assumption underlying this as well as the twomphase model is that the influence of a scalar field $\phi$ can be approximated by a cosmological 'constant' which decays instantaneously to zero at some instant $t_{f}$. This is a very strong restriction on the form

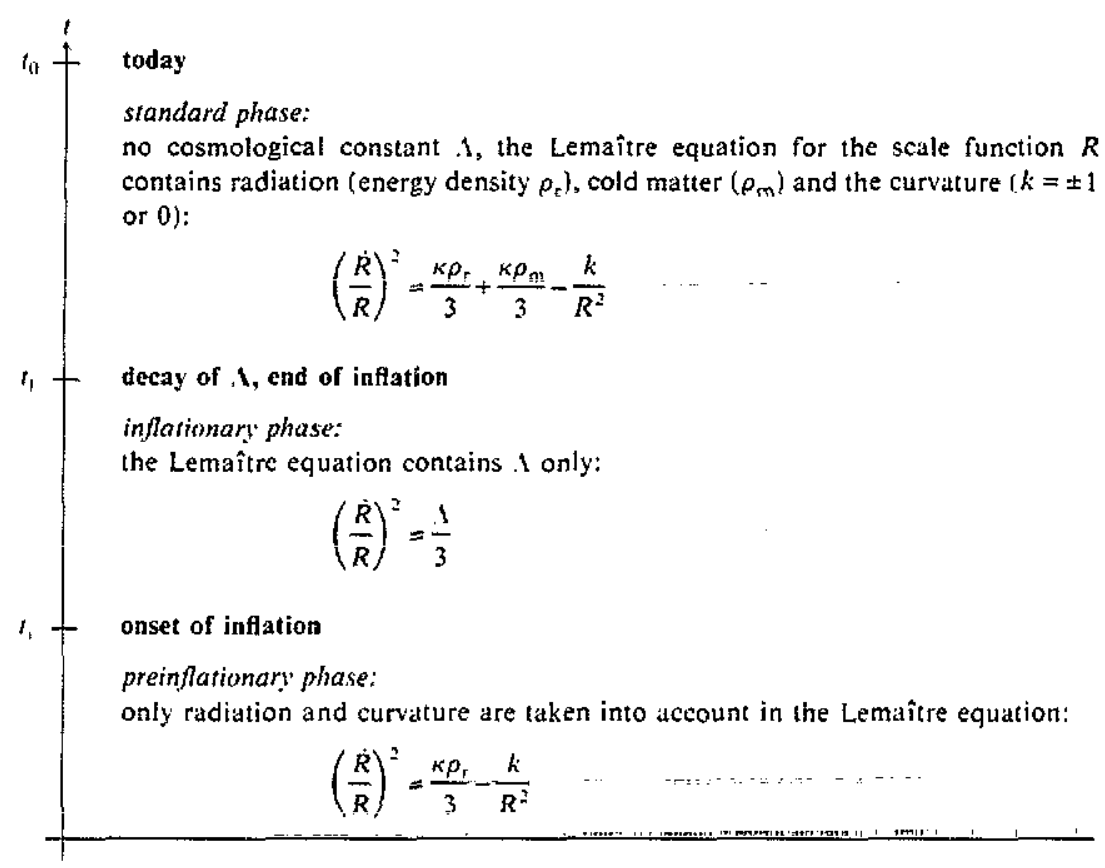

Figure 1. Ellis model. 
of the potential of the scalar field $\phi$ (for a discussion of this point in the new inflationary scenario in a flat RW model, see [7]) and is furthermore based on the assumption that the coupling between scalar field and radiation can be neglected in the slow-rolling period which depends on the form of the coupling (see [1] for a 'good' coupling and appendix 2 for a "bad' coupling).

Another, fictitious, discontinuity in $\ddot{R}$ at the onset of inflation $t_{\mathrm{i}}$ was introduced by Ellis only to simplify the calculations. It does not represent a phase transition, but it does affect the choice of parameters of the model.

Since $R$ and $\dot{R}$ have to be continuous throughout (see appendix 1), the following equations have to hold ( $k / R_{\mathrm{f}}^{2}$ has been neglected; it is very small in comparison with $\left.k / R_{\mathrm{j}}^{2}\right)$ :

$$
\kappa \rho_{\mathrm{ri}}-3 \frac{k}{R_{\mathrm{i}}^{2}}=\kappa \rho_{\mathrm{tot} \mathrm{i}}=\Lambda .
$$

The 'radii' of the primeviāi and višual horizons are given by

$$
u_{\mathrm{d}}=\int_{0}^{t_{\mathrm{d}}} \frac{\mathrm{d} t}{R(t)} \quad u_{\mathrm{v}}=\int_{t_{\mathrm{d}}}^{t_{\mathrm{t}}} \frac{\mathrm{d} t}{R(t)}
$$

where $t_{\mathrm{d}}$ is the time of decoupling when $R_{0} / R_{\mathrm{d}} \approx 10^{3}$. (The roman indices $\mathrm{m}$ and $\mathrm{r}$ refer to cold matter and radiation, the indices $\mathrm{i}, \mathrm{f}, \mathrm{d}$ and 0 stand for 'at $t_{\mathrm{j}}$ ', 'at $t_{\mathrm{f}}$ ', 'at $t_{\mathrm{d}}$ ' and 'at $t_{0}$ ', respectively).

The requirement that the horizon problem be resolved leads to a relation between $\Omega_{\mathrm{i}}$ and $R_{\mathrm{f}} / R_{\mathrm{i}}$ for given $R_{\mathrm{o}} / R_{\mathrm{f}}, \Omega_{\mathrm{m} 0}$ and $\Omega_{\mathrm{r} 0}$. The allowed values of $\Omega_{\mathrm{i}}$ are restricted to a small range and therefore the duration of the inflationary phase cannot vary significantly. Ellis obtained in the, in view of observations, most interesting, low-density case $\Omega<1$ :

$$
u_{\mathrm{d}}>10 u_{\mathrm{v}} \Leftrightarrow-\frac{1}{2} \ln \Omega_{\mathrm{i}}+\ln 2>u_{\mathrm{v}} .
$$

The amount of inflation $\left(R_{\mathrm{f}} / R_{\mathrm{i}}\right)$ has to obey

$$
27-\log \frac{R_{0}}{R_{\mathrm{f}}}+\log \frac{R_{\mathrm{f}}}{R_{\mathrm{i}}}=25-\log \left[0.48\left(1+\Omega_{\mathrm{i}}\right)\right] .
$$

Since in a hyperbolic model $1+\Omega_{i} \in\left[1,2\left[\right.\right.$ (actually $\Omega_{j} \ll 1$, see [6]) we have

$$
-1.98+\log \frac{R_{0}}{R_{f}} \leqslant \log \frac{R_{\mathrm{f}}}{R_{i}}<-1.68+\log \frac{R_{0}}{R_{\mathrm{r}}} .
$$

One expects a larger inflation (larger $R_{\mathrm{t}} / R_{\mathrm{i}}$ ) to solve the horizon problem too, and therefore to give a possible model. For a too large inflation (small $R_{\mathrm{i}}$ ), equation (1) cannot be fulfilled since $k \rho_{\mathrm{rt}}+\left(3 / R_{\mathrm{i}}^{2}\right) \geqslant\left(3 / R_{\mathfrak{i}}^{2}\right)>A \approx \kappa \rho_{\text {tot }}$. This problem does not arise in the two-phase model, which lacks the discontinuity at $t_{\mathrm{i}}$.

\section{The two-phase model}

\subsection{The general model}

Lemaître's equation

$$
\begin{aligned}
\left(\frac{\dot{R}}{R}\right)^{2} & =\frac{\kappa}{3}\left(\rho_{\mathrm{r}}+\rho_{\mathrm{m}}\right)-\frac{k}{R^{2}}+\frac{\Lambda}{3} \\
& =\frac{\kappa}{3}\left[\rho_{\mathrm{r}(1)}\left(\frac{R_{0}}{R}\right)^{4}+\rho_{\mathrm{m}, 1}\left(\frac{R_{0}}{R}\right)^{3}\right]-\frac{k}{R^{2}}+\frac{\Lambda}{3}
\end{aligned}
$$


for an interactionless mixture of radiation and dust can be solved explicitly in terms of elementary functions if either $\rho_{m}=0$ or $\Lambda=0$. We therefore improve Ellis' model by taking two phases only, separated by the 'true' phase transition at $t_{1}$. where $\Lambda$ jumps from its positive primordial value to its present value, assumed to vanish. In the 'early' phase I, $\rho_{m}=0$; in the "late" phase II, $A=0$. Again $R$ and $\dot{R}$ are to be continuous throughout, with $\ddot{R}$ discontinuous only at $t_{f}$ (see appendix 1 ). Thus, we consider the model illustrated in figure 2 .

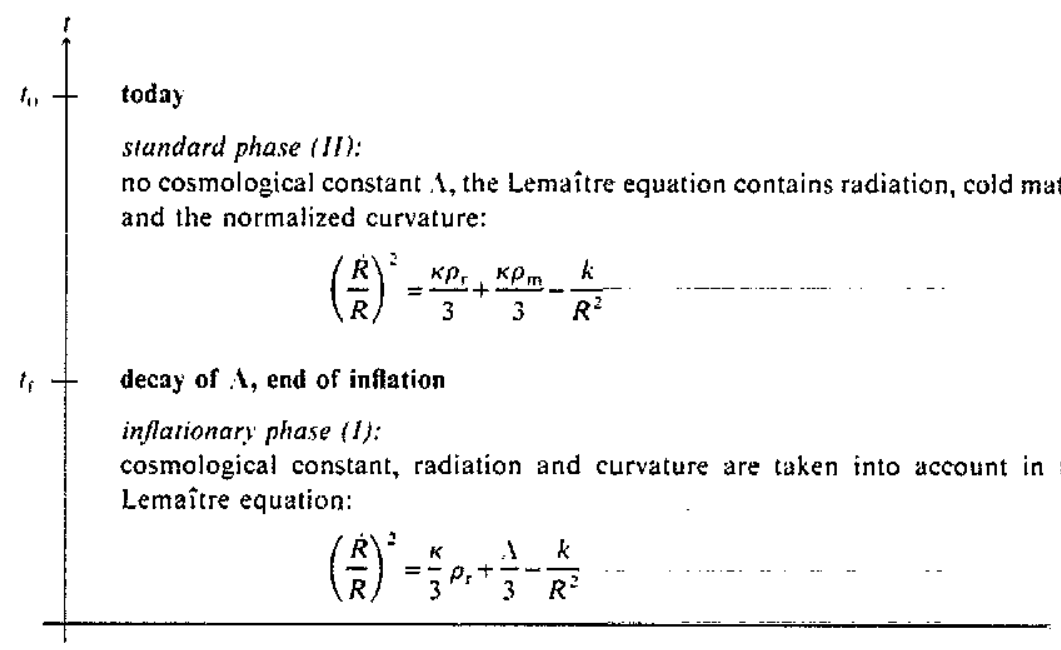

Figure 2. The two-phase model.

Because of the junction condition, the energy densities in phase 1 and II are not independent. We have:

$$
\rho_{\mathrm{rrll}}+\rho_{\mathrm{mrll}}=\rho_{\mathrm{rfl}}+(\Lambda / \kappa)
$$

( $\rho_{\mathrm{rfl} / \mathrm{ll}}$ stands for the limit of $\rho_{\mathrm{r}}$ for $t \rightarrow t_{\mathrm{r}}$ in phase I/II). Thus we have only the freedom to distribute the energy between the energy of the 'false vacuum' $\rho_{1}:=\Lambda / \kappa$ and the energy of the radiation $\rho_{11}$, described by the parameter $\omega$ :

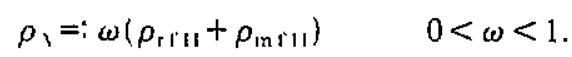

This parameter $\omega$ also measures the degree of dominance of vacuum energy over radiation energy which begins shortly after $R_{\mathrm{i}}$ where $\rho_{\mathrm{l}}=\rho_{\mathrm{r}}\left(R_{\mathrm{i}}\right)$, and ends at $R_{\mathrm{r}}$ with the decay of the cosmological constant:

$$
\frac{\rho_{1}}{\rho_{r i l}}=\frac{\omega}{1-\omega}=\left(\frac{R_{i}}{R_{t}}\right)^{4}
$$

Note the difference between our definition of the duration of inflation and the usual one in terms of the slow-rolling of the scalar field. In a more detailed description of a scalar field our whole phase I would correspond to slow-rolling. Nevertheless we do not have exponential expansion all the time; exponential expansion starts shortly after the instant when $\rho_{1}=\rho_{\mathrm{r}}-3 k / \kappa R^{2}$ ( $R_{\mathrm{i}}$ as defined above is convenient for computations\}. Before $t_{i}$ it does not matter how we approximate the contribution of the scalar field to the evolution equation, which is dominated by radiation and curvature. 
Choosing $\omega$ suitably allows one to solve the horizon problem.

As we want to construct an inflationary model for realistic values of observable quantities we write the Lemaitre equation (2) for phase II in terms of the parameters $\Omega_{\mathrm{r} 0}, \Omega_{\mathrm{m} 0}, H_{0}, R_{\mathrm{o}}$ and $k$ :

$$
\dot{R}^{2} R^{2}=-k R^{2}+\Omega_{\mathrm{m} 0} H_{0}^{2} R_{0}^{3} R+\Omega_{\mathrm{ru}} H_{0}^{2} R_{0}^{4} .
$$

These parameters are constrained by the equations

$$
R_{0}^{2}:=R^{2}\left(t_{0}\right)=\left\{\begin{array}{ll}
\frac{k}{\left(\Omega_{0}-1\right) H_{0}^{2}} & k \neq 0 \\
\frac{1}{H_{0}^{2}} & k=0
\end{array} \quad\left(\Omega_{0}=\Omega_{\mathrm{m} 0}+\Omega_{\mathrm{r}(0)}\right)\right.
$$

the second of which is a convention, and by

$$
\Omega_{0}\left\{\begin{array}{l}
< \\
>
\end{array}\right\} 1 \Leftrightarrow k=\left\{\begin{array}{c}
-1 \\
0 \\
1
\end{array}\right.
$$

For phase I the Lemaître equation (2) is

$$
\dot{R}^{2} R^{2}=\omega \xi R_{\mathrm{f}}^{-2} R^{4}-k R^{2}+(1-\omega) \xi R_{\mathrm{f}}^{2} .
$$

The symbol $\xi$ stands for

$$
\xi= \begin{cases}\frac{\Omega_{\mathrm{r} 0}}{\left|1-\Omega_{0}\right|} \frac{R_{0}^{2}}{R_{\mathrm{f}}^{2}}+\frac{\Omega_{\mathrm{m} 0}}{\left|1-\Omega_{0}\right|} \frac{R_{\mathrm{o}}}{R_{\mathrm{r}}} & k= \pm 1 \\ \Omega_{\mathrm{ro}} \frac{R_{0}^{2}}{R_{\mathrm{f}}^{2}}+\Omega_{\mathrm{m} 0} \frac{R_{\mathrm{u}}}{R_{\mathrm{f}}} & k=0\end{cases}
$$

$\xi$ is related to the total energy density $\rho_{\text {tut } \mathrm{f}}$ at the beginning of phase 11 by

$$
\kappa \rho_{\text {tot }}=3 R_{\mathrm{f}}^{-2} \xi
$$

A lower bound for $R_{\mathrm{t}} / R_{\mathrm{f}}$ arises from the assumption that the baryons have been created in the CP-violating decay of the $\mathrm{X}$ particles after inflation; an upper bound derives from the energy density at which one expects to observe quantum gravity effects. Its order-of-magnitude value $R_{v} / R_{f}=10^{27}\left(T \approx 10^{14} \mathrm{GeV}\right.$, see $\left.[2,8]\right)$ is quite uncertain because it is based on heuristic arguments only since there is no accepted, let alone confirmed, grand unified theory. We shall refer to the dependence of the two-phase models on this uncertainty later.

To construct a particular model we start with $\Omega_{\mathrm{mo}}$ and $\Omega_{\mathrm{ru}}$ (always taken as 0.0004 ); these values determine $H_{0} R_{0}$ and $k$. Thus it is possible to calculate the (dimensionless) radius of the visual horizon. The primeval particle horizon $u_{\mathrm{a}}$ (bounded below by $\left.u_{1}:=\int_{0}^{t_{1}}\left(1 / R_{1}(t)\right) \mathrm{d} t\right)$ is larger than $u_{\mathrm{v}}$ provided $\omega$ is sufficiently close to 1 . To determine the admissible values and to examine whether they are similar to the ones in the flat case is the next task.

\subsection{Computation of the $\omega$-ranges which solve the horizon problem}

The solutions of (4) are well known the parametrized form $\left(R / R_{0}\right)(\tau)$ with $\tau$ given by $\tau=\int_{0}^{t}\left(1 / R\left(t^{\prime}\right)\right) \mathrm{d} t^{t}$ (see, for example, [9]). The inequality

$$
u_{\mathrm{d}}=\int_{11}^{t_{\mathrm{s}}} \frac{1}{R_{\mathrm{II}}(t)} \mathrm{d} t \ll u_{\mathrm{s}}=\int_{t_{\mathrm{t}}}^{t_{1}} \frac{1}{R_{\mathrm{II}}(t)} \mathrm{d} t
$$


which expresses the horizon problem of the standard model, implies that the visual horizon of the two-phase model is approximated by $\tau_{0}=\int_{0}^{t}\left(1 / R_{11}(t)\right) \mathrm{d} t$.

To solve (5) we introduce $Z=\left(R / R_{\mathrm{f}}\right)^{2}$ and $\tilde{t}:=2 \sqrt{\omega \xi} R_{\mathrm{f}}^{-1} t$ and get

$$
\left(\frac{\mathrm{d} Z}{\mathrm{~d} \tilde{i}}\right)^{2}=Z^{2}-\frac{k}{\omega \xi} Z+\frac{1-\omega}{\omega} .
$$

This looks like (4) with $k=-1$ after the substitution $R(t) \rightarrow R[\tau]$. The general solution has the form $a(\cosh \tilde{t}-c)+b \sinh \tilde{t}$. In positively curved universes there can be a big bounce. This is not possible for solutions of (4) where one always has a big bang. The constants $a, b$ and $c$ are determined by the differential equation, the continuity of $R(t)$ and $\dot{R}(t)$ at $t_{\mathrm{f}}$ and the choice of the parameter $\omega$.

If $k=0$ or $k=-1, \mathrm{~d} Z / \mathrm{d} \tilde{t}$ is always greater than 0 and therefore these models start with a big bang.

The solutions are

$$
R_{1}(\tilde{t})= \begin{cases}R_{\mathrm{f}}\left[\left(\frac{1-\omega}{\omega}\right)^{1 / 2} \sinh \tilde{t}\right]^{1 / 2} & k=0 \\ R_{\mathrm{i}}\left[\frac{1}{2 \omega \xi}(\cosh \tilde{t}-1)+\left(\frac{1-\omega}{\omega}\right)^{1 / 2} \sinh \tilde{t}\right]^{1 / 2} & k=-1 .\end{cases}
$$

In the spherical case, $k=1$, five possibilities arise. We only give the solutions. In the subsequent discussion we do not pay any attention to this case, for in case ( $v$ ) with the largest amount of inflation there is a bounce, and therefore no horizon problem arises. All models which are compatible with the observed age of the universe are allowed (i.e. $\Omega_{0} \leqslant 1.5$ for $\Lambda_{0}=0$, see [10]).

The classification criteria for these cases are the existence and positions relative to $R_{\mathrm{f}}$ of the solutions of the equation $(\mathrm{d} Z / \mathrm{d} \tilde{t})^{2}=0$. These criteria decide whether there is a bounce or not. See also the qualitative drawings of the solutions in figure 3 .
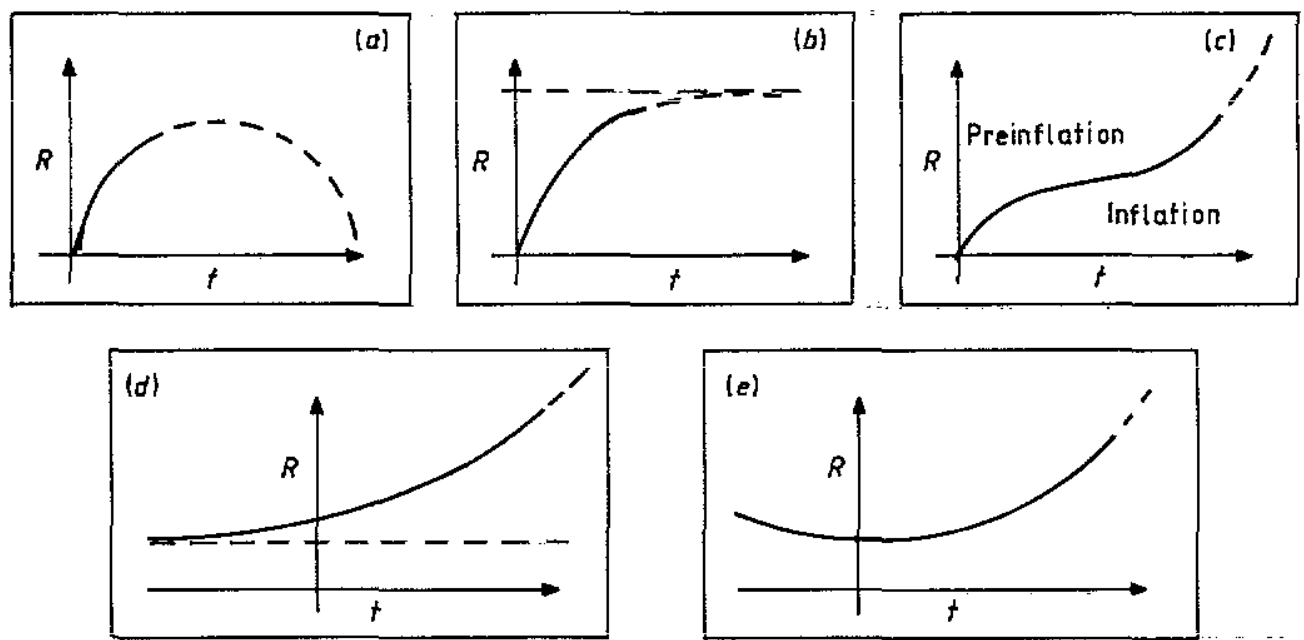

Figure 3. $R_{t}(t)$ for the five spherical cases. Solid curves refer to $t \leqslant t_{1}$, broken curves-not part of our model-refer to $t \geqslant t_{1}$. 
(i) $0 \stackrel{\text { def }}{\leqslant} \omega \leq \frac{1}{2}\left(1-\sqrt{1-\xi^{-2}}\right)(\Rightarrow(1 / 2 \omega \xi)>\sqrt{(1-\omega) / \omega})\left(Z_{1,2}\right.$ are two distinct solutions and $R_{t}^{2}<Z_{1,2}$, no bounce)

$$
R_{\mathrm{l}}(\tilde{t})=R_{\mathrm{r}}\left[-\frac{1}{2 \omega \xi}(\cosh \tilde{t}-1)+\left(\frac{1-\omega}{\omega}\right)^{1 / 2} \sinh \tilde{t}\right]^{1 / 2}
$$
bounce)

(ii) $\left.\omega=\frac{1}{2}\left(1-\sqrt{1-\xi^{-2}}\right) \quad \Leftrightarrow(1 / 2 \omega \xi)=\sqrt{(1-\omega) / \omega}\right) \quad\left(Z_{1}=Z_{2}\right.$ and $R_{\mathrm{f}}^{2}<Z_{1,2}$, no

$$
R_{\mathrm{I}}(\tilde{t})=R_{\mathrm{f}}\left[\left(\frac{1-\omega}{\omega}\right)^{1 / 2}\left(1-\mathrm{e}^{-i}\right)\right]^{1 / 2}
$$

(iii) $\frac{1}{2}\left(1-\sqrt{1-\xi^{-2}}\right)<\omega<\frac{1}{2}\left(1+\sqrt{1-\xi^{-2}}\right) \quad(\Rightarrow(1 / 2 \omega \xi)<\sqrt{(1-\omega) / \omega}) \quad\left(Z_{\mathrm{t}, 2}\right.$ do not exist, no bounce)

$$
R_{\mathrm{l}}(\tilde{t})=R_{f}\left[-\frac{1}{2 \omega \xi}(\cosh \tilde{t}-1)+\left(\frac{1-\omega}{\omega}\right)^{1 / 2} \sinh \tilde{t}\right]^{1 / 2} .
$$

(iv) $\omega=\frac{1}{2}\left(1+\sqrt{1-\xi^{-2}}\right) \quad(\Rightarrow(1 / 2 \omega \xi)=\sqrt{(1-\omega) / \omega}) \quad\left(Z_{1}=Z_{2} \quad\right.$ and $\quad R_{i}^{2}>Z_{1,2}$, no bounce)

$$
R_{1}(\tilde{t})=R_{\mathrm{r}}\left[\left(1-\frac{1}{2 \omega \xi}\right) \mathrm{e}^{\tilde{t}}+\frac{1}{2 \omega \xi}\right]^{1 / 2}
$$

$\left(\tilde{t}\left(R_{\mathrm{f}}\right) \stackrel{!}{=} 0\right.$, there is a bounce at $\left.\tilde{t} \downarrow-\infty\right)$.

(v) $\omega>\frac{1}{2}\left(1+\sqrt{1-\xi^{-2}}\right)(\Rightarrow(1 / 2 \omega \xi)>\sqrt{(1-\omega) / \omega})\left(Z_{1,2}\right.$ are two distinct solutions and $R_{\mathrm{f}}^{2}>Z_{1.2}$, there is a bounce at $\tilde{t}=0$. For $(1-\omega) \downarrow 0$ this bounce occurs at $R \gg l_{\mathrm{p}}$ )

$$
R_{1}(\tilde{t})=R_{\mathrm{f}}\left(\frac{1}{2 \omega \xi}\left(\sqrt{1-4 \omega(1-\omega) \xi^{2}} \cosh \tilde{t}+1\right)\right)^{1 / 2} .
$$

It is not possible to give an explicit elementary expression for

$$
u_{1}:=\int_{\text {r(begin })}^{r_{r}} \frac{1}{R_{1}(t)} \mathrm{d} t
$$

since $u_{1}$ is an elliptic function. But for realistic values of $\Omega_{\mathrm{mo}}$ it is possible to find useful lower bounds (see (6) below).

Numerical results for some $\Omega_{\mathrm{mo}}$ are

$\begin{array}{llll}\Omega_{\mathrm{m}(1}= & 0.3 & 0.6 & 0.9996\left(\Omega_{\mathrm{il}}=1\right) \\ (1-\omega) \leqslant & 5.6 \times 10^{-103} & 7.7 \times 10^{-1113} & 10^{-101} \\ \log \left(R_{f} / R_{\mathrm{i}}\right) \geqslant & 25.6 & 25.5 & 25.3\end{array}$

Therefore no significant modification is necessary to solve the horizon problem in non-flat Friedmann universes as compared with flat ones.

But in the data we started from there was one with a large uncertainty, the parameter $R_{0} / R_{f}$. What happens to the model when we choose a different value? One gets the (at first sight) surprising answer: the value of the scale function at $t_{i}$ does not have to vary for solving the horizon problem when $R_{0} / R_{\mathrm{t}}$ is in the range $R_{0} / R_{\mathrm{f}}=10^{27 \pm 6}$. For $\Omega<1$ and a high inflation rate $\left(\frac{1}{4}>\omega(1-\omega) \xi^{2}\right)$ we get from $a(\cosh t-1)+b \sinh t \leqslant$ $\frac{1}{2}(a+b)\left(\mathrm{e}^{\prime}-1\right)$ :

$$
\frac{2}{\sqrt{1+2 \sqrt{\omega(1-\omega)} \xi}} \tan ^{-1}\left[\left(\frac{\frac{1}{2}+\omega \xi}{\underline{1}+\sqrt{\omega(1-\omega)} \xi}-1\right)^{1 / 2}\right] \leqslant u_{1} \text {. }
$$

For the range of $R_{0} / R_{\mathrm{l}}$ values considered, $10^{38} \leqslant \xi \leqslant 10^{\text {i2 }}$ and $(1-\omega) \ll 1$, we can set $\tan ^{-1}[\ldots] \approx \pi / 2$. Therefore the lower limit stays constant if $(1-\omega) \sim \xi^{-2} \sim R_{\mathrm{t}}^{-4}$, 
and thus $R_{\mathrm{i}} \sim R_{\mathrm{r}}(1-\omega)^{1 / 4}=$ constant. Variation of $R_{0} / R_{\mathrm{f}}$ leads to a variation of $\rho_{\text {tot } \mathrm{f}}$ and so to a variation of $A$. This causes a different expansion rate and compensates the change in the amount of inflation.

This fact implies that the model is essentially unaffected by a quantum gravity phase of whatever kind. It is generally believed that such a theory is necessary for a typical variation scale of the metric comparable with the Planck length $l_{p}$. In a $(\Omega<1)$-RW universe the scale of variation of the metric is roughly $R$. So we cannot say anything about the contribution of the quantum era to $u_{\mathrm{J}}$. But this does not change much in the two-phase model, since for $R<R$, the scale function $R$ behaves like that of a pure radiation model (the influence of $\Lambda$ can be neglected). Further, for the upper bound of the allowed values of $(1-\omega)$ we obtain $R_{\mathrm{i}} \approx 10^{\times} l_{1}$ and, because of the horizon problem,

$$
\int_{1)}^{R_{1}} \frac{1}{R_{\text {class }}(t)} \mathrm{d} t \ll \int_{R_{\mathrm{p}}}^{R_{1}} \frac{1}{R(t)} \mathrm{d} t
$$

The quantum-era contribution to $u_{1}$ is not significant and the horizon problem is solved in any kind of quantum gravity theory.

If one does not want to restrict $(1-\omega)$ by the above consideration such that $R_{1} \gg I_{\mathrm{P}}$, one can make $u_{1}$ infinite because $u_{1}(\omega)$ diverges for $\omega \uparrow 1$.

Another important point is the relation between $\Omega_{0}$ and the value $\Omega_{h}$ of $\Omega$ at a time $t_{h}$ at which we choose initial values (flatness problem). The definition of

$$
\Omega:=\frac{\kappa \rho}{3 H^{2}}=1+\frac{k}{\dot{R}^{2}}
$$

and equation (5) yield

$$
\Omega(R)=\frac{\omega \xi\left(R / R_{f}\right)^{2}+(1-\omega) \xi\left(R_{t} / R\right)^{2}}{\omega \xi\left(R / R_{f}\right)^{2}+(1-\omega) \xi\left(R_{t} / R\right)^{2}-k} \quad \cdots-R<R_{i} .
$$

The local minimum iñ phase $\mathrm{I}$ is

$$
\Omega=\frac{2 \sqrt{(1-\omega) \omega} \xi}{2 \sqrt{(1-\omega) \omega} \xi-k}
$$

for $R=R_{\mathrm{i}}$. For $R \downarrow 0, \Omega$ quickly tends to 1 . In the model for which $\Omega_{\mathrm{m} n}=0.3$ and $(1-\omega)$ is maximal the density parameter $\Omega\left(R_{\mathrm{i}}\right)$ is 0.46 . For smaller $(1-\omega), \Omega_{\mathrm{i}}$ decreases very fast.

The relation between $\Omega_{0}$ and $\Omega_{h}$ depends strongly on $\omega$. We do not think that it is possible to determine $R_{\mathrm{t}} / R_{f}$ (or $\xi$ ) and 1 sufficiently accurately to make any statement about $\Omega_{h}$. For nearly all $\Omega_{h}$ in ]0, 1[ with $R_{b}<R_{i}\left((1-\omega)_{m \alpha x}\right)$ it is possible to solve the horizon problem and to get any observed $\Omega_{0}$, by a proper choice of $(1-\omega)$.

For the qualitative behaviour of $\Omega(R)$ see also figure 4 .

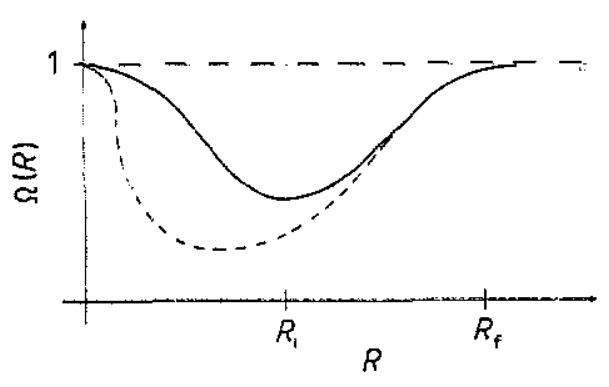

Figure 4. Plots of $\{2(R)$ for different sets of conditions. The solid curve is for $\Omega_{\mathrm{nnt1}}=0.3$ and $(\mathrm{i}-\omega)=$ $(1-w)_{\operatorname{mal}}$. The broken curve is for $\Omega_{\mathrm{m}, 1}=0.3$ and $(1-\omega)<(1-w)_{\min }$. 
If we fix the amount of inflation $\left(R_{\mathrm{f}} / R_{\mathrm{i}}\right)$ we can use (7) and the corresponding equation for phase II,

$$
\Omega\left(R_{0}\right)=\frac{\Omega_{\mathrm{rr}}\left(R_{\mathrm{f}} / R_{0}\right)^{2}+\Omega_{\mathrm{mr}}\left(R_{\mathrm{f}} / R_{0}\right)}{\Omega_{\mathrm{rl}}\left(R_{\mathrm{f}} / R_{0}\right)^{2}+\Omega_{\mathrm{ml}}\left(R_{\mathrm{f}} / R_{0}\right)+1-\Omega_{\mathrm{r}}}
$$

to determine the relation between a probability measure for $\Omega$ on an initial value surface characterized by $R_{\mathrm{b}}$ and the probability measure for $\Omega_{0}$.

\section{Deviations from the idealization of the two-phase model}

For deviations from the two-phase model it is in general not possible to solve the evolution equations, but nevertheless it is often possible to compare the horizons of two models without knowing the solutions to the evolution equations.

Let the horizons of two models be determined through

$$
\tilde{u}=\int_{t_{\mathrm{i}}}^{\tilde{r}^{\prime}} \frac{1}{\tilde{R}(t)} \mathrm{d} t \text { and } u=\int_{t_{\mathrm{i}}}^{t^{\prime}} \frac{1}{R(t)} \mathrm{d} t
$$

with strictly increasing functions $R$ and $\tilde{R}$ which could mean, for example, two maximal expansion rates in an anisotropic model. Then $u$ and $\tilde{u}$ are the minimal extensions of the horizons. Changing integration variables we find

$$
u=\int_{R\left(t_{i}\right)}^{R\left(t^{\prime}\right)} \frac{\mathrm{d} R}{R \cdot \dot{R}[R]}
$$

and

$$
\begin{aligned}
\tilde{u} & =\int_{\tilde{R}\left(\tilde{t}_{i}\right)}^{\tilde{R}\left(\tilde{t}^{\prime}\right)} \frac{\mathrm{d} R}{R \cdot \hat{R}[R]} \\
& =\int_{\tilde{R}\left(\tilde{t}_{\mathrm{i}}\right)}^{\tilde{R}\left(\tilde{\mathrm{f}}^{\prime}\right)} \frac{\mathrm{d} R}{R \cdot \dot{R}[R]} \underbrace{\frac{\dot{R}[R]}{\tilde{R}[R]}}_{*} .
\end{aligned}
$$

If $\tilde{R}\left(\tilde{t}_{\mathrm{i}}\right)=R\left(t_{\mathrm{i}}\right)$ and $\tilde{R}\left(\tilde{t}^{\prime}\right)=R\left(t^{\prime}\right)$, we can estimate $\tilde{u}$ in terms of $u$ if we can estimate the expression *. An important example consists of two models with RW metrics. Then $R$ and $\tilde{R}$ are the scale functions and the evolution equations are Lemaittre equations:

$$
\dot{R}=f(R) \quad \dot{\hat{R}}=g(\tilde{R}) .
$$

Then the ratio $*$ is simply

$$
*=\frac{f(R)}{g(R)} \text {. }
$$

\subsection{A general form of matter in phase $I$}

We allow in phase I arbitrary perfect fluids with $0 \leqslant p \leqslant \frac{1}{3} \rho$.

$\nabla_{\triangleleft} T^{a b}=0$ yields

$$
\rho \sim \frac{1}{R^{3+\varepsilon(1)}} \quad 0 \leqslant \varepsilon(t) \leqslant 1 .
$$


We compare such a model with a two-phase model ( ) with the same total energy density $\tilde{\rho}_{i^{\prime \prime l}}=\rho_{\mathrm{fll}}$ and the same Hubble constant at $t_{\mathrm{f}}$.

The solution of the horizon problem for the two-phase model yields a value $(1-\tilde{\omega})$. Let us choose $\tilde{\omega}$ in such a way that the horizon problem is solved very well.

The term $*$ in equation $(8)$ is

$$
\left(\frac{(\kappa / 3) \tilde{\rho} R^{2}+(\Lambda / 3) R^{2}-k}{(\kappa / 3) \rho R^{2}+(\Lambda / 3) R^{2}-k}\right)^{1 / 2} \leqslant 1
$$

Using the mean value theorem we conclude that there is a number $c$ in the interval ]0, 1 [ such that $u=c \hat{u}$. Choosing a large enough $\tilde{u}$, it should be possible to solve the horizon problem for the 'twiddled' model under consideration. A proof has to take into account that $c$ is a function of $\tilde{\omega}$, but we feel that it is not worthwhile to provide such a proof.

\subsection{The Bianchi type I as an example of an anisotropic model}

For a Bianchi type I model one can choose coordinates in such a way [11] that

$$
\bar{g}_{a x \beta}=\left(\begin{array}{cccc}
-1 & & & \\
& X^{2}(t) & & \\
& & Y^{2}(t) & \\
& & & Z^{2}(t)
\end{array}\right) .
$$

$X(t), Y(t)$ and $Z(t)$ are deterhingęd through the differential system

$$
\begin{aligned}
& A+\frac{1}{2}(\rho-p)=\frac{\ddot{X}}{X}+\frac{\dot{X} \dot{y}}{X Y}+\frac{\dot{X} \dot{Z}}{X Z}=\frac{\ddot{Y}}{Y}+\frac{\dot{X} \dot{Y}}{X Y}+\frac{\dot{Y} \dot{Z}}{Y Z}=\frac{\ddot{Z}}{Z}+\frac{\dot{X} \dot{Z}}{X Z}+\frac{\dot{Y} \dot{Z}}{Y Z} \\
& \rho+A=\frac{\dot{X} \dot{Y}}{X Y}+\frac{\dot{X} \dot{Z}}{X Z}+\frac{\dot{Y} \dot{Z}}{Y Z} \quad \text { (first integral) } \\
& \frac{\dot{\rho}}{\rho+p}=-(\ln X Y Z) .
\end{aligned}
$$

With the substitution [12]

$$
\begin{array}{ll}
X(t)=R(t) \mathrm{e}^{t(t)} & Y(t)=R(t) \mathrm{e}^{\beta(t)} \\
Z(t)=R(t) \mathrm{e}^{\gamma(t)} & \alpha+\beta+\gamma=0
\end{array}
$$

the system acquires the form

$$
\begin{aligned}
& 3\left(\frac{\dot{R}}{R}\right)^{2}-1=\kappa \rho+\left(3 c_{1}^{2}+c_{2}^{2}\right) R^{\cdots} \quad \dot{\alpha}=\left(c_{1}+c_{2}\right) R^{-3 .} \\
& \dot{\beta}=\left(c_{1}-c_{2}\right) R^{-3 .} \quad \frac{\dot{\rho}}{\rho+p}=-3(\ln R(t)) .
\end{aligned}
$$

$c_{1}$ and $c_{2}$ are constants which are determined by the initial values. The comoving coordinates should be chosen in such a way that

$$
\lim _{i \rightarrow \infty}(\alpha, \beta, \gamma)=0
$$


In the analysis of equation (9) something very surprising appears. Suppose we have a phase where $\Lambda$ dominates (9). Then $\rho$ is 'inflated away'. The same happens with the anisotropy term $\mathscr{A}:=\left(3 c_{1}^{2}+c_{2}^{2}\right) R^{-6}$. We reach a RW-like phase and can surely solve the horizon problem. But there remains no anisotropy, except one created in the decay of the fluctuating scalar field, which should not have the discrete symmetry of a Bianchitype model [13]. Thus discrete symmetries in the angular dependence of the microwave background radiation would disprove inflation.

Since the other Bianchi types differ only by different couplings of the scale functions $X, Y, Z$ governing the anisotropic expansion, the effect should be similar.

So if we live in a Bianchi model other than the Rw case, inflation is hardly possible.

In any case, inflation would have to explain an anisotropy in the background radiation by the decay of the scalar field. Survival of primordial fluctuations is not possible.

\section{Conclusion}

In this work we have given a model for inflation in a non-flat $\mathrm{R} w$ universe with radiation at all times. The requirement 'solution of the horizon problem' yields an upper limit for the ratio of radiation energy to radiation energy plus false vacuum energy (cosmological constant), denoted $(1-\omega)$, equation (3). This limit is equivalent to a lower bound for the duration of the inflationary period, which starts when the cosmological constant begins to dominate the radiation energy. Also we have seen that the density parameter $\Omega$ can have values clearly different from 0 and 1 .

Since every model contains simplifications we have discussed in section 4 the influence of some deviations from the two-phase model.

One referee pointed out that the significant lower bound for the amount of inflation may be due to the fact that the observed fluctuations in the microwave background, related to the quantum fluctuations of the scalar field, are extremely small [7]. In the 'worked example' of this paper for the potential $V=V_{0}-\beta \phi^{3}+\lambda \phi^{4}$ the authors have found that $\lambda$ has to be smaller than a certain value depending on the microwave background fluctuations and that $V_{0}$ and $\beta$ depend on $\lambda$ only. This can be translated into a condition on $V_{0}$, which is roughly $\Lambda / \kappa$ in our model. Our $\Lambda / \kappa$ fulfills that condition, but we do not know to what extent one may apply that constraint, which is based on fluctuation calculations on a de-Sitter background metric (see references in [7]), to different backgrounds since curvature and radiation as well as the cosmological constant influence the evolution. Furthermore the model yielding the fluctuation spectrum used in [7] has weak points (see [14]).

Whether properties of fluctuations restrict-or possibly even disprove-our model can be decided only by investigating such fluctuations, a task which can be tackled now based on the background model.

\section{Acknowledgment}

PH would like to thank all the members of the General Relativity group of the Max-Planck-Institut für Astrophysik (München) for discussions and encouragement during his Diplomarbeit. 
Appendix 1. General junction conditions at spacelike hypersurfaces

Let $\Sigma$ be a hypersurface given by $f\left(x^{a}\right)=0$. Let us write

$$
[A]:=\lim _{1 \downarrow 0} A-\lim _{1 \uparrow 0} A=A_{+}-A_{-}
$$

for the jump discontinuity of any quantity which is continuous at each side of $\Sigma$.

Because Einstein's field equation requires the Ricci tensor $R_{\mathrm{ab}}$ to be well defined, we need at least piecewise continuous Christoffel symbols, and therefore a continuous metric $g_{a l}\left(\left[g_{a b}\right]=0\right)$. Otherwise, $R_{a b}$ would contain products of $\delta$-like distributions which are not well defined.

The field equation then yields an energy-momentum-tensor $T_{u b}$ of the form

$$
r_{a b}=S_{a h} \delta(f)+\tilde{T}_{a b}
$$

with $\tilde{T}_{a h}$ piecewise continuous.

Papapetrou and Hamoi [15] have shown that $S_{u b}$ is "tangential" to $\Sigma$, i.e. that

$$
S_{a h} N^{a}=0
$$

where $N^{a}=g^{a t} \nabla_{h} f$ is normal to $\Sigma$. In the case where $\Sigma$ is spacelike (phase transition in cosmology) we can introduce observers with 4-velocity $N^{a}$. These observers would measure a surface energy density

$$
S_{00}=S_{a h} N^{a} N^{b}=0
$$

on $\Sigma$. The dominant energy condition $\left(S_{00} \geqslant\left|S_{a b}\right|\right)$ then yields

$$
S_{a h}=0
$$

which implies, according to the calculations of [15], that the extrinsic curvatures of $\Sigma$ with respect to both of its sides, must be equal to each other.

In $\mathrm{RW}$ models the continuity of the metric and the extrinsic curvature of the time-slices are equivalent to the constancy of the curvature index and the continuity of $R(t)$ and $\dot{R}(t)$. The argument given here both strengthens and simplifies previous arguments given by Ellis [16].

\section{Appendix 2. Two examples of 'bad' coupling}

In this chapter we will give two examples of how a 'strange' coupling between a scalar field and radiation can prevent the development of an inflationary phase. We do not claim to present a physically realistic model, we only want to stress that coupling may be important.

Let us start with a Klein-Gordon field with the Lagrangian

$$
\mathscr{L}=-\frac{1}{2}\left(\nabla^{\prime \prime} \phi \nabla_{n} \phi\right)-V(\phi)
$$

and corresponding energy-momentum tensor

$$
T_{a h}^{b}=\nabla_{a} \phi \nabla_{b} \phi-\frac{1}{2} g_{a b}\left(\nabla^{\prime} \phi \nabla_{c} \phi+2 V(\phi)\right) \text {. }
$$

Since we restrict the further calculations to a $\mathrm{RW}$ model whose comoving observers have 4-velocity $u^{\text {ct }}$, we may assume spatial homogeneity and get

$$
T_{s h}^{s_{k}}=\left(\rho_{\phi}+p_{s}\right) u_{u} u_{b}+p_{s,} g_{u h}
$$


with

$$
\rho_{\phi}=\frac{1}{2} \dot{\phi}^{2}+V(\phi) \quad \rho_{\phi}=\frac{1}{2} \dot{\phi}^{2}-V(\phi) .
$$

For $|\dot{\phi}|^{2} \ll|2 V(\phi)|$ (slow-rolling) we have $p \approx-\rho$ and therefore the energy-momentum tensor looks like a cosmological constant $\Lambda$ multiplied by the metric $g_{a} b$. Adding radiation ( $p=\frac{1}{3} \rho$, energy-momentum tensor $T_{a b}^{\mathrm{r}}$ ) to this model yields

$$
\begin{aligned}
& 3 H^{2}=\frac{\kappa}{2}\left(\dot{\phi}^{2}+2 V(\phi)\right)+\kappa \rho_{\mathrm{r}}-3 \frac{k}{R^{2}} \\
& \underbrace{\dot{\rho}_{\mathrm{r}}+4 \rho_{\mathrm{r}} \frac{\dot{R}}{R}} u_{a} \nabla_{b} T_{\mathrm{r}}^{u b}+\underbrace{\dot{\phi}\left(\ddot{\phi}+\frac{\mathrm{d} V}{\mathrm{~d} \phi}\right)+\dot{\phi}^{2} \frac{\dot{R}}{R}} u_{a} \nabla_{h} T_{\phi}^{a h}=0
\end{aligned}
$$

as evolution equations. To uniquely determine the solutions $R(t), \phi(t)$ and $\rho_{\mathrm{r}}(t)$ we need to specify the coupling between $\rho_{\mathrm{r}}$ and $\phi$. Albrecht et al [1] have shown by numerical calculations that a coupling

$$
u_{a} \nabla_{b} T_{r}^{a h}=\text { constant } \times \dot{\phi}^{d} \phi^{\varsigma-2 d}=-u_{a} \nabla_{b} T_{\phi}^{a h}
$$

gives the desired cosmological-constant-like behaviour of $T_{\phi \phi}^{a b}$. But this is a property of this special kind of coupling. Counterexamples for a 'good' behaviour are as follows.

(i) The loss in the energy density of $\phi$ is the source for $\nabla_{a} T_{\mathrm{r}}^{a b}$ :

$$
\dot{\rho}_{\mathrm{r}}+4 \rho_{\mathrm{r}} \frac{\dot{R}}{R}=-\frac{\mathrm{d} \rho_{t}}{\mathrm{~d} t}=-\dot{\phi}\left(\ddot{\phi}+\frac{\mathrm{d} V}{\mathrm{~d} \phi}\right) .
$$

Equation (10) then reduces to

$$
\dot{\phi}=\frac{\dot{R}}{R}=0
$$

with the only solution $\dot{\phi}=0 . \phi$ can only change if there is a discontinuity in the evolution of the universe, for example a sudden decay of the scalar field. The difficulties (see [17]) arising then have led to the search for new models (new inflation, chaotic inflation).

(ii) The change in the potential $V$ of the scalar field is the source for $\nabla_{a} T_{s}^{u t h}$ :

$$
\dot{\rho}_{\mathrm{r}}+4 \rho_{\mathrm{r}} \frac{\dot{R}}{R}=-\frac{\mathrm{d} V}{\mathrm{~d} t}=-\dot{\phi} \frac{\mathrm{d} V}{\mathrm{~d} \phi} .
$$

Equation (10) then reduces to

$$
\dot{\phi} \ddot{\phi}+3 \dot{\phi}^{2} \frac{\dot{R}}{R}=0
$$

with the solutions $\dot{\phi}=0$ and $\dot{\phi} \sim 1 / R^{3}$. The first is the same as (i), and the second does not match with new or chaotic inflation. In these pictures there should be a small change in $\phi$ for small $R$.

Thus we conclude that the type of coupling between radiation and a Klein-Gordon field may be an essential factor for an inflationary phase to occur. It may essentially influence the duration of the slow-rolling phase ( $e$-folds of inflation). 


\section{References}

[1] Albrecht A, Steinhardt P J. Turner M S and Wilczek F 1982 Reheating an inflationary universe Phys. Rev. Lett. 48 1437-40

[2] Guth A H 1981 Inflationary universe: A possible solution to the horizon and flatness problems $P h y s$. Rev. D 23 347-56

[3] Blau S K and Guth A H 1987 Inflationary cosmology 300 Years of Gravitation ed S W Hawking and W' Israel (Cambridge: Cambridge University Press) pp 524-603

[4] Abbott L F and So-Young Pi (eds) 1986 Inflationary Cosmology (Singapore: World Scientific)

[S] Goldwirth D S and Piran T $19 \overline{9} \overline{0}$ Inhomogeneity and the onset of inflation Phy's. $\bar{R} e v$. Left. $64 \overline{2} \overline{8} \overline{5} \overline{2}-\overline{5}$

[6] Ellis G F R 1988 Does inflation necessarily imply $\Omega=1$ ? Class, Quantum Grax. 5 891-901

[7] Steinhardt P J and Tumer M S 1984 Prescription for successful new inflation Phys. Rev. D 29 2162-71

[8] Linde A D 1989 Particle Physics and Cosmology 25sh Int. Conf. in High Energy Physics (Munich) ed R Kotthaus and J H Kühn (Berlin: Springer) pp 356-86

[9] Ellis G F R 1987 Standard cosmology Vth Brazilian School of Cosmology and Gravitation ed M Novello (Singapore: World Scientific) pp 83-151

[10] Ehlers J and Rindler W 1989 A phase-space representation of Friedmann-Lemaître universes containing both dust and radiation and the inevitability of a big bang Mon. Not. R. Astron. Soc, 238 503-21

[11] Ellis G F R and MacCallum M A H 1969 A class of homogeneous cosmological models Commun. Math. Phy's 12 108-41

[12] Jacobs K C 1968 Spatially homogeneous and Euclidian cosmological models with shear Astrophys. J. $153661-78$

[13] Schmidt B G 1969 Discrete isotropies in a class of cosmological models Commun. Math. Phy.s. 15 329-36

[14] Boucher W and Traschen J 1988 Semiclassical physics and quantum fluctuations Phys. Rev. D 37 3522-32

[15] Papapetrou A and Hamoi A 1968 Couches simples de matière en relativité générale Ann. Inst. Henri Poincaré 9 179-211

[16] Ellis G F R 1987 Note on 'Symmetry changes in Friedmann cosmologies' by S S Bayin Astrophys. J. 314 1-2

[17] Guth A H and Weinberg E J 1983 Could the universe have recovered from a slow first-order phase-transition? Nucl. Phys. B $312321-64$ 